\title{
Protective Effect of Long Term Administration of Gum Arabic on Oxidative Stress in Hepatic Tissue of Diabetic Rats
}

\author{
Mohammed Babiker ${ }^{1,4}$, Mohammed Elimam Ahamed Mohammed ${ }^{2,3}$, Mohammed Amanullah*4, Tarig \\ Abbas $^{1}$ and Mutaz Siddig Dafallah ${ }^{5}$
}

${ }^{1}$ Faculty of Medicine, University Of Al Neelain, Sudan

${ }^{2}$ Department of Chemistry, King Khalid University, Saudi Arabia

${ }^{3}$ Sudan Atomic Energy Commission, Sudan

${ }^{4}$ Department of Clinical Biochemistry, King Khalid University, Saudi Arabia

${ }^{5}$ Department of Physiology, King Khalid University, Saudi Arabia

Received: May 12, 2018; Published: May 24, 2018

*Corresponding author: Mohammed Amanullah, Associate Professor, Department of Clinical Biochemistry, College of Medicine, King Khalid University, Greiger, Abha-61431, Saudi Arabia

\begin{abstract}
Activity of key antioxidant enzymes plays a critical role in the induction of hyperglycemia related tissue damage. Gum Arabic (GA) is an edible, dried sticky exudate from Acaciaseyal and Acacia Senegal and is rich with non-viscous soluble fiber. It is commonly used in food industry and pharmaceutical field as an emulsifier and preservative. It has strong antioxidant properties, and used to reduce the experimental nephrotoxicity against gentamicin, cisplatin and to ameliorate cardiotoxicity. However, the effects of GA on oxidative stress in liver of type I diabetic rats have not been reported. Whether GA can change oxidative related genes expression in liver of type I diabetic rat remains less clear. In the present study, we used type I diabetic rat model to investigate our hypothesis that supplementation of GA in drinking water may protect liver by reducing oxidative damage. 60 male Sprague-Dawley rats were housed and Type I diabetes mellitus (DM) was induced by a single intraperitonial injection (i.p.) of stereptozotocin STZ (65 mg/kg).
\end{abstract}

The rats were anaesthetized with pentobarbital sodium and liver removed and processed for biochemical measurements viz. Thiobarbituric acid reactive substances (TBARS) an index of malondialdehyde production and antioxidant enzymes. Key antioxidant enzymes including SOD, CAT and Glutathione of rat liver were measured. The antioxidant activity as a function of oxidative stress in hepatic tissue in the six groups obtained for the levels of reduced glutathione (GSH) in the livers of gum arabic treated group of rats were significantly different when compared to control group Furthermore this was significantly different in rats with DM treated with Insulin and gum Arabic. Diabetic rat group showed significant decreases in all antioxidant enzymes activities when compared to the control. Significant increase of hepatic MDA concentrations in diabetic group compared to the control was observed. Q.PCR was used to measure antioxidant enzymes wherein mRNA expression in the liver was seen. Stereptozotocin induced diabetes caused significant decrease in liver SOD mRNA expression. However, the treatment of GA significantly increased mRNA expression of hepatic SOD compared to diabetic group or those treated with insulin. Furthermore mRNA significantly increased and reached on par with control in diabetes rats treated with insulin and GA. We concluded that the GA treatment reduced lipid peroxidation, improved the activities of antioxidant enzymes and their mRNA expression in the liver of diabetic rats. Thus, GA may be useful in reducing oxidative stress.

Keywords: Diabetes Mellitus; Liver damage; Antioxidants; Gum Arabic

Abbreviations: DM: Diabetes Mellitus; ROS: Reactive Oxygen Species; HSP70: Heat Shock Protein 70; GA: Gum Arabic; NAFLD: Non-Alcoholic Fatty Liver Disease; TBARS: Thiobarbituric Acid Reactive Substances; SOD: Superoxide Dismutase

\section{Introduction}

Action of key cell reinforcement catalysts assumes a basic part in the acceptance of hyperglycemia related tissue harm [1] Oxidative pressure initiated by the lopsidedness of oxidants/cancer prevention agents harm of organic macromolecules, including starches, proteins, lipids, and nucleic acids, cause aggravations in cell homeostasis and generation of other receptive atoms that reason more damage[2]. The significance of oxidative pressure and its association with the pathology of diabetes mellitus (DM) alongside 
related intricacies have been broadly investigated $[1,3]$. Past examinations announced that the generation of reactive oxygen species (ROS) in diabetes starts the improvement of perpetual diabetic sores on blood vessels [4], Retina [5], kidneys [6], and neurodegenerative diseases [7]. DM is a constant and most regular metabolic issue that has turned out to be pandemic in the twenty first century [8]. Around 347 million individuals were influenced with DM in 2011 overall [9].

The World Wellbeing Association predicts that diabetes will be the seventh driving reason for death in2030. Oxidative worry in DM causes a few unfavorable consequences for the cell physiology [10]. It diminished glutathione (GSH) level in diabetes [11], diminished catalyze activity [12], down managed renal SOD [13] and expanded heat shock protein 70 (HSP70) level in patients with type2 DM [14]. Oxidative pressure has been accounted for as a key factor in the beginning of pathogenesis and diabetic complexities [15]. Clinical and test ponders suggested that the liver might be affected by DM in the long haul [16-18]. Histological highlights of fatty liver disease initiated by DM and non-alcoholic fatty liver disease (NAFLD) can't be recognized from ethanol-instigated hepatic steatosis [18]. Gum Arabic (GA) is a consumable, dried sticky exudate from Acaciaseyal and Acacia Senegal is rich with non-thick solvent fiber. It is usually utilized as a part of nourishment industry and pharmaceutical field as an emulsifier and additive [19].

In North Africa and Middle East, it's utilized as an oral cleanliness specialist by different groups for a few centuries [20]. GA is utilized as a part of Arabic society pharmaceutical to diminish both recurrence and need of hemodialysis in incessant renal failure patients [21]. It has solid cancer prevention agent properties, and used to decrease the test nephrotoxicity against gentamicin [21], cisplatin [22] and to improve cardiotoxicity [23]. Be that as it may, the impacts of GA on oxidative worry in liver of type I diabetic rats have not been accounted for. Regardless of whether GA can change oxidative related qualities articulation in liver of type I diabetic rodent stays less clear. Consequently, in the present examination, we utilized type I diabetic rodent model to explore our speculation that supplementation of GA in drinking water may secure liver by lessening oxidative harm, and the decrease of oxidative harm might be related with balance of liver oxidative related qualities articulation.

\section{Rational of the Study}

In the present study we aim to use a diabetic rat model, to test whether adding GA to insulin therapy would ameliorate the diabetic induced changes in liver function, and whether the action is mediated by an effect on oxidative stress markers.

\section{Material and Methods}

\section{Animals}

A total of 60 male Sprague-Dawley rats (age 5-6 weeks) weighing $200 \pm 10$ g were included in this study. Animals were housed in the animal house a controlled environment with a 12:12-hrs light/ dark cycle. Animals were acclimatized for one week before the study and had free access to water and standard rat chow throughout the experimental period.

\section{Animal Groups}

This study followed a randomized controlled animal experimental design. The 60 male Sprague-Dawley rats included in this study were divided into 6 groups $(\mathrm{n}=10$ each) as follows:

\section{A. Control groups:}

a) Non- treated control (C): rats were injected intraperitoneally once with citrate buffer only.

b) Gum Arabic treated control (GAC): rats were injected intraperitoneally with buffer as "C" group and received 10\% w/v of Gum Arabic in drinking water.

\section{B. Experimental Groups:}

a) None treated diabetic group (D): diabetic group neither treated with insulin nor GA.

b) Diabetic + insulin (DI): Rats received insulin only.

c) Diabetic + GA (DGA): rats received GA only after induction of diabetes.

d) Diabetic + insulin + GA (DIGA): rats received insulin and GA after induction of diabetes.

\section{Induction of Diabetes Mellitus (DM)}

Type I diabetes mellitus (DM) was induced as described by [24]. Briefly, a single intraperitonial injection (i.p.) of stereptozotocin STZ (65 mg/kg) in freshly prepared citrate buffer (0.1 M, pH 4.5) was given after an overnight fasting. DM was verified by measuring blood glucose in surviving rats after 3 days, through tail/neck blood sampling. Rats with non-fasting blood glucose level of $\geq 20 \mathrm{mmol} / \mathrm{L}$ after $48 \mathrm{~h}$ of STZ injection were considered to be diabetic [25]. The experimental period was 12 weeks, a period which has been proven to induce detectable diabetic complications in the liver [26].

\section{Tissue Preparation}

The rats were anaesthetized with pentobarbital sodium (60 $\mathrm{mg} / \mathrm{kg}$ body weight, intraperitoneally). Liver was removed, cleaned of gross adventitial tissue, blotted dry and processed for biochemical measurements. Tissue was homogenized in $50 \mathrm{mM}$ phosphate buffer (pH 7.4) using a Polytron homogenizer. The resultant supernatant was used for measurement of Thiobarbituric acid reactive substances (TBARS) and antioxidant enzymes. The TBARS levels, were measured as an index of malondialdehyde production and then lipid peroxidation was assessed in the tissues by the method of Yagi [27] as previously described [28].

\section{Evaluation Of Hepatic Antioxidant Enzyme Activity}

The Superoxide dismutase (SOD), glutathione, catalase (CAT) and glutathione (GSH) commercial reagents were purchased from Sigma Chemical Company, USA. Liver tissues (100 mg) were cut into small pieces and homogenized in ice-cold saline buffer $(0.85 \%, \mathrm{pH}$ 7.4) (1:9, wt/v) with an Ultra-Turrax (T8, IKA-labortechnik Staufen, Germany). Liver homogenates were centrifuged at $1000 \times \mathrm{g}$ for 15 $\min$ at $4^{\circ} \mathrm{C}$, and the supernatants were collected. The supernatants were used for the assays of SOD, Glutathione, CAT and GSH. The an- 
tioxidative status of liver was estimated by measuring the level of different antioxidants in the liver. The activity of SOD was measured according to the method of [29]. The activities of Glutathione and CAT were measured by the methods described by [30], and [31] respectively. All assays were measured with the clinical chemistry assay kits according to the manufacturer's recommended procedure. The antioxidant activity as a function of oxidative stress in hepatic tissue was determined according to the manufacturers information; catalase (abcam- ab83464 catalase assay kit- Colorimetric/ Fluorometric), Superoxide dismutase (Cayman chemical- Superoxide Dismutase assay kit- item number. 706002), malondailadehyde (specialized kit from Cayman Chemical. Michigan, USA) and glutathione (SIGMA- ALDRICH- glutathione assay kit- catalog number CS0260). The obtained results were analyzed by SPSS version 20. The mean values of the parameters of the two groups were analyzed using t- test.

\section{RNA Extraction and Real-Time PCR}

About 100 mg of liver was ground in liquid N2, and a portion of about $50 \mathrm{mg}$ was used for RNA extraction using TRIzol total RNA kit (Invitrogen, Biotechnology Co., Ltd., Carlsbad, CA, USA) according to the manufacturer's instruction. Two approaches were taken to ensure that all the total RNA preparations are free of genomic DNA contamination. First, total RNAs were treated with $10 \mathrm{U}$ DNase I (RNase Free, D2215, Takara, Japan) for $30 \mathrm{~min}$ at $37^{\circ} \mathrm{C}$, and puri- fied according to the manufacturer's protocol. Second, the primers for the reference gene ( $\beta$-actin) were designed to span an intron, so any genomic DNA contamination could be reported easily with an extra product in the melting curves for real-time PCR. Real-time PCR was performed in Mx3000P (Stratagene, USA) according to the previous publications $[32,33]$. Primers specific for SOD, and $\triangle \mathrm{GCL}$ (Table 1) was synthesized by Geneary (Shanghai, China), and rat $\beta$-actin was used as a reference gene for normalization purpose. The method of $2-\Delta \Delta \mathrm{Ct}$ was used to analyze the real-time PCR data [34]. The mRNA abundances were presented as the fold change relative to the average level of the control group.

\section{Ethical Clearance}

This study was conducted after academic and ethical approval from the College of Medicine, King Khalid University, Abha, Saudi Arabia".

\section{Statistical Analysis}

Descriptive statistics was performed to check the normality and homogeneity of variances before using parametric analyses. Data was analyzed using SPSS statistical program. Values are expressed as frequency, percentage and mean \pm SD. Testing significance was performed using $\chi 2$ test and the one-way analysis of variance (ANOVA). P-values $\leq 0.05$ were considered statistically significant.

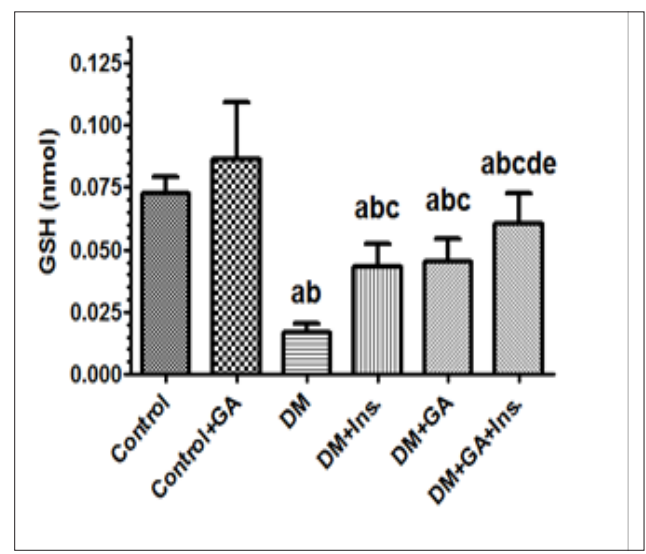

Figure 1: Levels of reduced glutathione (GSH) in the livers of all experimental groups of rats. Values (mean \pm SD) were obtained for 10 animals/group. a: Significantly different when compared to control group. b: Significantly different when compared to control+GA. c: Significantly different when compared DM d: significantly different when compared to DM+Ins. e:significantly different when compared to DM+GA. Ins: insulin. GA: Gum Arabica.

\section{Results}

\section{Effect of GA on Hepatic Antioxidant Enzymes Activities}

Key antioxidant enzymes including SOD, CAT and Glutathione of rat liver were measured. The antioxidant activity as a function of oxidative stress in hepatic tissue in the six groups is presented in Figure 1 to 3 . Values (mean \pm SD) obtained for the levels of reduced glutathione (GSH) in the livers of gum arabic treated group of rats were significantly different when compared to control group. Furthermore there were also significantly different in rats with DM treated with Insulin and gum Arabic (Figure 1). Diabetic rat group showed significant decreases in all antioxidant enzymes activities when compared to the control. Levels of total superoxide dismutase (SOD) in the livers of experimental groups of rats showed a diverse tendency wherein rats with diabetes mellitus did not show any significant increase in SOD upon treatment with gum arabic however addition of insulin with gum Arabic to diabetic rats increased SOD very significantly (Figure 2). Finally the levels of catalase (CAT) showed very significant increase in diabetes rats treated either with insulin or gum Arabic alone or in combination (Figure 3). However, the treatment of GA significantly $(\mathrm{P}<0.05)$ increased the antioxidant enzymes activities including SOD, CAT and Glutathione compared to that of control and diabetic groups. 


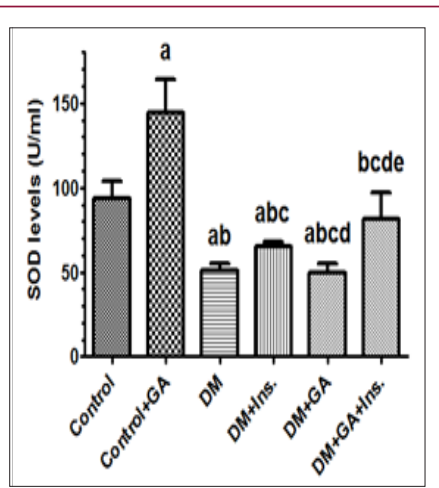

Figure 2: Levels of total superoxide dismutase (SOD) in the livers of all experimental groups of rats. Values (mean $\pm S D)$ were obtained for 10 animals/group. a: Significantly different when compared to control group. b: Significantly different when compared to control+GA. c: Significantly different when compared DM d: significantly different when compared to DM+Ins. e:significantly different when compared to DM+GA. Ins: insulin. GA: Gum Arabica.

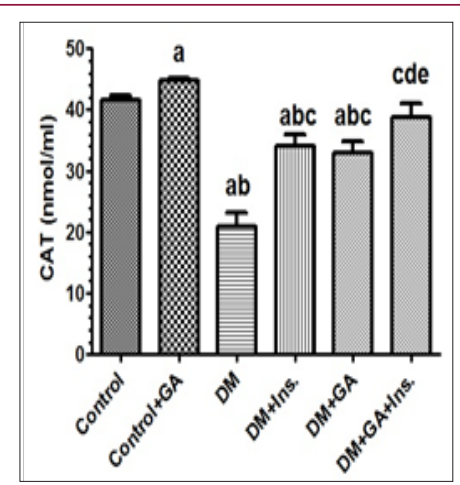

Figure 3: Levels of catalase (CAT) in the livers of all experimental groups of rats. Values (mean \pm SD) were obtained for 10 animals/group. a: Significantly different when compared to control group. b: Significantly different when compared to control+GA. c: Significantly different when compared DM d: significantly different when compared to DM+Ins. e:significantly different when compared to DM+GA. Ins: insulin. GA: Gum Arabica.

\section{Effect of GA on Hepatic Lipid Peroxidation}

Malondialdehyde (MDA) as a biomarker of oxidative stress is routinely used to evaluate the extent of lipid peroxidation. In the present study, we observed significant increases of hepatic MDA concentrations in diabetic group compared to the control. However, the treatment of GA significantly $(\mathrm{P}<0.05)$ decreased MDA concentrations compared to that of diabetic rat group (Figure 4). In addition, the treatment of GA significantly increased liver GSH concentration compared to the control and diabetic rat groups. The levels of thiobarbituric reactive acid substances (GSH) in the livers of all experimental groups of rats showed a similar trend, however their levels were significantly higher in diabetic rats treated with gum Arabic and insulin (Figure 4).

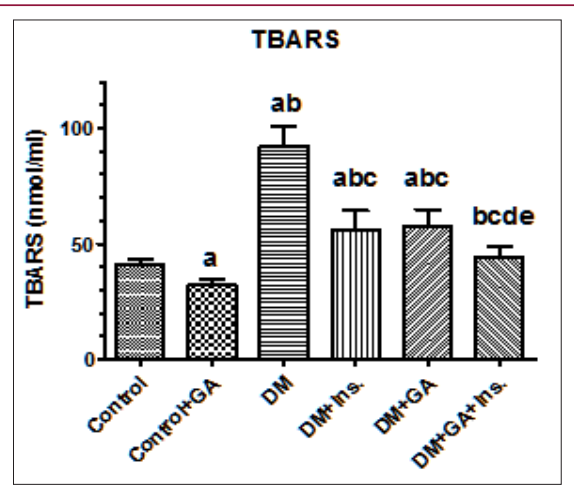

Figure 4: Levels of thiobarbituric reactive acid substances (TBARS) in the livers of all experimental groups of rats. Values (mean \pm SD) were obtained for 10 animals/group. a: Significantly different when compared to control group. b: Significantly different when compared to control+GA. c: Significantly different when compared DM d: significantly different when compared to DM+Ins. e:significantly different when compared to DM+GA. Ins: insulin. GA: Gum Arabica. 


\section{Effect of GA on hepatic antioxidant genes expression.}

Q.PCR was used to measure antioxidant enzymes mRNA expression in the liver. Stereptozotocin induced diabetes caused significant decrease in liver SOD mRNA expression. However, the treatment of GA significantly increased mRNA expression of hepatic SOD (Figure 5) compared to diabetic group or those treated with insulin. Furthermore mRNA significantly increased and reached on par with control in diabetes rats treated with insulin and GA. A similar trend was seen with $₫ \mathrm{GLC}$ mRNA expression in relation to $\beta$-actin mRNA, wherein treatment of GA to diabetes induced rats significantly increased its expression and it was much more significantly increased when GA treated in combination with insulin (Figure 6).

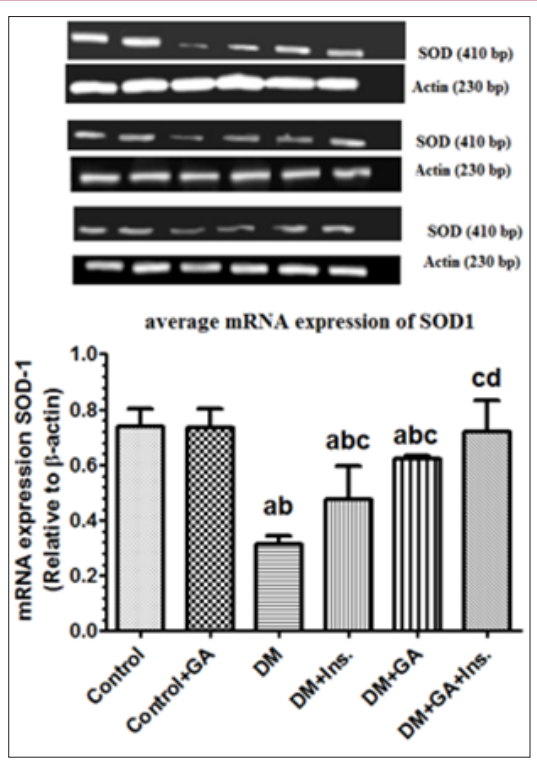

Figure 5: mRNA levels of SOD in the livers of all experimental groups of rats. Values (mean $\pm S D$ ) were obtained for 3 animals / group. a: Significantly different when compared to control group. b: Significantly different when compared to control+GA. c: Significantly different when compared DM d: significantly different when compared to DM+Ins... L: Ladder; sample 1: Control; Sample 2: Control+ Gum Arabica (GA); Samples 3: Diabetes Mellitus (DM); sample 4: DM+Insulin; Sample 5: DM+GA; Sample 6: DM+insulin+GA Sample 7 is a negative control.

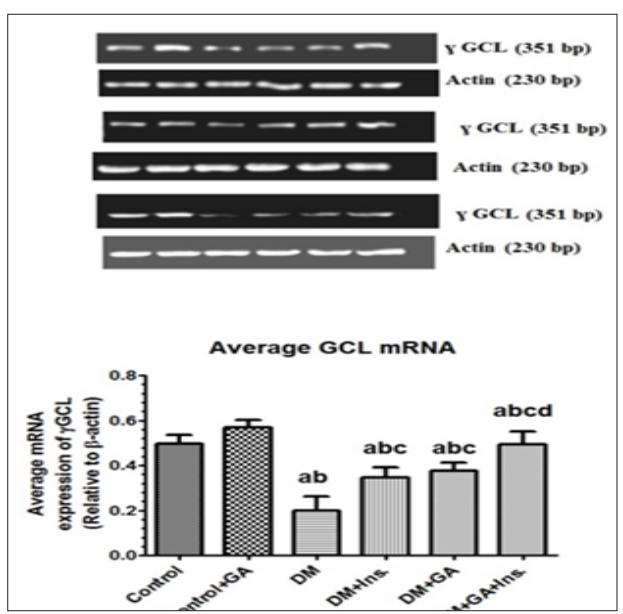

Figure 6: mRNA levels of $y$ GCL in the livers of all experimental groups of rats. Values (mean \pm SD) were obtained for 3 animals/group. a: Significantly different when compared to control group. b: Significantly different when compared to control+GA. c: Significantly different when compared DM d: significantly different when compared to DM+Ins... L: Ladder; sample 1: Control; Sample 2: Control+ Gum Arabica (GA); Samples 3: Diabetes Mellitus (DM); sample 4: DM+Insulin; Sample 5: DM+GA; Sample 6: DM+insulin+GA.

\section{Discussion}

A vast group of confirmation proposes a noteworthy part of oxidative stress being developed and advancement of diabetic difficulties. Liver function tests are as often as possible utilized as a part of clinical practice to screen liver issue and to check the devel- opment of known disorders [35]. We might want to exceptionally specify that it's hard to control the quantity of GA extract dispensation through drinking water. Superoxide dismutase (SOD) [36], catalase (CAT) [37], and glutathione [38] are viewed as the most essential protection components against responsive oxygen metabolites which embroiled in the oxidative harm [10,39]. 
All the antioxidant parameters incorporated into our examination were fundamentally expanded. The emphatically influenced parameters were the glutathione, TBARS, superoxide dismutase, and catalase. Like our discoveries Abdelkareem A. Ahmed and his exploration bunch said that gum Arabic essentially expanded superoxide dismutase, catalase, glutathione peroxidase and GSH They likewise inferred that the gum Arabic dosage altogether diminished the MDA [40]. Treatment of male rats with Trichloroacetate diminished the level of hepatic superoxide dismutase and catalase and expanded the grouping of MDA. The trichloroactate poisonous quality was turned around by gum Arabic and peppermint oil $[41,42]$. Ali BH led trial to research antioxidant activity of three distinct doses of gum Arabic on kidney and liver of rats. He quantified GSH and superoxide dismutase and his outcomes showed the nonappearance of a critical impact of gum Arabic on the two parameters [43].

In the present investigation, the treatment of Stereptozotocin caused huge reductions in activities of SOD, CAT, and Glutathione, related with huge increments in malondialdehyde (MDA) creation that could reflect oxidative pressure; which might be a mirror to diminished antioxidant defense potential[10,43]. In any case, the treatment of GA fundamentally expanded action of SOD, CAT and Glutathione in the liver. GA may act either straightforwardly by rummaging the receptive oxygen metabolites, as a result of the nearness of different cell reinforcement mixes [19,44,45], or through expanding the formation of antioxidant molecules. The component of activity by which GA enhances antioxidant capacity could be because of the way that GA contains amino acid residues, for example, lysine, tyrosine and histidine, which are for the most part considered as cell reinforcements molecules [46,47]. Besides, the antioxidant successes of GA in organic frameworks require a more straightforward learning of the cell antioxidant capacity [19,48-51].

Expanded oxidative stress with weakened antioxidant defense framework is believed to be the crucial variables prompting the pathogenesis and diabetic complexities. In the present examination, we utilized Q.PCR to see if unfaltering state interpretation levels were adjusted. The treatment of GA altogether expanded mRNA articulation of SOD and $\triangle \mathrm{GCL}$. The expansion in $\triangle \mathrm{GCL}$ and SOD mRNA together with the expansion in their activities may propose the association of post-translational modification in changing the activities of these catalysts [52].

\section{Conclusion}

We concluded that the GA treatment reduced lipid peroxidation, improved the activities of antioxidant enzymes and their mRNA expression in the liver of diabetic rats. Thus, GA may be useful in reducing oxidative stress. Further investigations are required to find out the effects of GA in diabetic model.

\section{References}

1. Y Wu, L Tang, B Chen (2014) Oxidative stress: implications for the development of diabetic retinopathy and antioxidant therapeutic perspectives. Oxid MedCell Longev 1: 752387.

2. E Birben, UM Sahiner, C Sackesen, S Erzurum, O Kalayci (2012) Oxidative stress and antioxidant defense. World Allergy Organ J 5: 9-19.
3. I Afanas'ev (2010) Signaling of reactive oxygen and nitrogen species in Diabetesmellitus. Oxid Med Cell Longev 3: 361-373.

4. SM Son (2012) Reactive oxygen and nitrogen species in pathogenesis of vascular complications of diabetes. Diabetes Metab J 36: 190-198.

5. Y Zhao, K Yang, F Wang, Y Liang, Y Peng, et al. (2012) Associations between metabolic syndrome and syndrome components and retinal microvascular signs in a rural Chinese population: the Handan Eye Study. Graefes Arch Clin Exp Ophthalmol 250: 1755-1763.

6. T Bondeva, G Wolf (2014) Reactive oxygen species in diabetic nephropathy: friend or foe. Nephrol Dial Transplant.

7. LR Borza (2014) A review on the cause-effect relationship between oxidative stress and toxic proteins in the pathogenesis of neurodegenerative diseases. Rev Med Chir Soc Med Nat Iasi 118: 19-27.

8. SA Tabish (2007) Is Diabetes Becoming the Biggest Epidemic of the Twenty-first Century? Int J Health Sci (Qassim) 1: V-VIII.

9. CD Mathers, D Loncar (2006) Projections of global mortality and burden of disease from 2002 to 2030. PLoS Med 3: e442.

10. BK Tiwari, KB Pandey, AB Abidi, SI Rizvi (2013) Markers of oxidative stress during diabetes mellitus, J. Biomarkers 2013: 1-8.

11. Y Dincer, T Akcay, Z Alademir, H Ilkova (2002) Assessment of DNA base oxidation and glutathione level in patients with type 2 diabetes. Mutat Res 505: 75-81.

12. L Goth (2000) Lipid and carbohydrate metabolism in acatalasemia. Clin Chem 46: 564-566.

13. C Wang, S Li, DJ Shang, XL Wang, ZL You, et al. (2011) Ant hyperglycemic and neuroprotective effects of one novel $\mathrm{Cu}-\mathrm{Zn}$ SOD mimetic. Bioorg Med Chem Lett 21: 4320-4324.

14. M Nakhjavani, A Morteza, L Khajeali, A Esteghamati O. Khalilzadeh, et al. (2010) Increased serum HSP70 levels are associated with the duration of diabetes. Cell Stress Chaperones 15: 959-964.

15. M Nakhjavani, A Morteza, AA Nargesi, E Mostafavi, A Esteghamati (2013) Appearance of leptin-HSP70 correlation, in type 2 diabetes. Meta Gene 1:1-7.

16. IA Leclercq, A Da Silva Morais, B Schroyen, N Van Hul, A Geerts (2007) Insulin resistance in hepatocytes and sinusoidal liver cells: mechanisms and consequences. J Hepatol 47:142-156.

17. LA Adams, P Angulo (2005) Recent concepts in non-alcoholic fatty liver disease. Diabet Med 22: 1129-1133.

18. SA Harrison, AM Diehl (2002) Fat and the liver-a molecular overview. Semin Gastrointest Dis 13: 3-6.

19. BH Ali, A Ziada G Blunden (2009) Biological effects of gum arabic: a review ofsome recent research, Food Chem Toxicol 47(1): 1-8.

20. VB Tyler, LJ Robbers (1977) Pharmacognosy. Lea \& Febiger. Philadelphia pp: 64-68.

21. AA Al-Majed, AM Mostafa, AC Al-Rikabi, OA Al-Shabanah (2002) Protective effects of oral arabic gum administration on gentamicininduced nephrotoxicity in rats. Pharmacol Res 46: 445-451.

22. AA Al-Majed, AR Abd-Allah, AC Al-Rikabi, OA Al-Shabanah, AM Mostafa (2003)Effect of oral administration of Arabic gum on cisplatin-induced nephrotoxicity in rats. J Biochem Mol Toxicol 17: 146-153.

23. AR Abd-Allah, AA Al-Majed, AM Mostafa, OA Al-Shabanah, AG Din, et al. (2002) Protective effect of arabic gum against cardiotoxicity induced by doxorubicin in mice: a possible mechanism of protection. J Biochem Mol Toxicol 16: 254-259.

24. AO Adeyi, BA Idowu, CF Mafiana, SA Oluwalana, OL Ajayi, et al. (2012) Rat model of food-induced non-obese-type 2 diabetes mellitus: comparative pathophysiology and histopathology. Int J Physiol Pathophysiol Pharmacol 4: 51-58. 
25. Szkudelski T (2001) The mechanism of alloxan and streptozotocin action in B cells of the rat pancreas. Physiol Res 50(6): 537-546.

26. G Kiran, CD Nandini, HP Ramesh, PV Salimath (2012) Progression of early phase diabetic nephropathy in streptozotocin-induced diabetic rats. Indian Journal of Experimental Biology 50: 133-140.

27. Ziyadeh FN, Han DC, Cohen JA, Guo J, Cohen MP (1998) Glycated albumin stimulates fibronectin gene expression in glomerular mesangial cells: Involvement of the transforming growth factor beta system. Kidney Int 53: 631-638.

28. Brownlee M (2001) Biochemistry and molecular cell biology of diabetic complications. Nature 414: 813-820.

29. M Minami, H Yoshikawa (1979) A simplified assay method of superoxide dismutase activity for clinical use. Clin Chim Acta 92: 337-342.

30. G Cohen, D Dembiec, J Marcus (1970) Measurement of catalase activity in tissue extracts. Anal Biochem 34: 30-38.

31. DE Paglia, WN Valentine (1967) Studies on the quantitative and qualitative characterization of erythrocyte glutathione peroxidase. J Lab Clin Med 70: 158-169.

32. AA Ahmed, HH Musa, JS Fedail, AZ Sifaldin, TH Musa (2015) Gum arabicdecreased visceral adipose tissue associated with down regulation of11-hydroxysteroid dehydrogenase type I in liver and muscle of mice. Bioact Carbohydr Dietary Fibre 6: 31-36.

33. HH Musa, AA Ahmed, TH Musa, JS Fedail (2015) Gum arabic downregulate PPAR- and SCD mRNA expression in mice. Pol Ann Med 22: 1117.

34. KJ Livak, TD Schmittgen (2001) Analysis of relative gene expression data usingreal-time quantitative PCR and the 2(-Delta Delta C(T)) Method. Methods 25: 402-408.

35. N Atasoy, A Erdogan, I Yalug, U Ozturk, N Konuk, et al. (2007) A review of liver function tests during treatment with atypical antipsychotic drugs: a chart review study. Prog. Neuro-Psychopharmacol. Biol. Psychiatry 31: $1255-1260$.

36. SS Gill, N Tuteja (2010) Reactive oxygen species and antioxidant machinery inabiotic stress tolerance in crop plants. Plant Physiol Biochem 48: 909-930.

37. M Tiedge, S Lortz, R Munday, S Lenzen (1998) Complementary action of antioxidant enzymes in the protection of bioengineered insulinproducing RINm5F cells against the toxicity of reactive oxygen species. Diabetes 47: 1578-1585.

38. CJ Tsai, CJ Hsieh, SC Tung, MC Kuo, FC Shen (2012) Acute blood glucose fluctuations can decrease blood glutathione and adiponectin levels in patients with type 2 diabetes. Diabetes Res Clin Pract 98: 257-263.
39. P Sharma, AB Jha, RS Dubey, M Pessarakli (2012) Reactive Oxygen species, oxidative damage, and antioxidative defense mechanism in plants under stressful conditions. J Bot 2012: 1-26.

40. Abdelkareem A Ahmed, Jaafar S Fedail, Hassan H Musa, Asghar Ali Kamboh, Amal Z Sifaldin, et al. (2015) Gum Arabic extracts protect against hepatic oxidative stress in alloxan induced diabetes in rats. Pathophysiology 22: 189-194.

41. Najla 0 Ayaz, Kholoud S Ramadan, Hoda EA Farid, Hanan S Alnahdi (2016) Protective role and antioxidant activity of arabic gum againsttrichloroacetate-induced toxicity in liver of male rats. Indian Journal of Animal Research.

42. Aly HM, Amal E Abd El-Kader, Amal M Abo El Maaty, Hafiza A Sharaf (2016) Andrological, oxidative stress and pathological effects of encapsulated peppermint oil with gum Arabic. Part 1 RJPBCS 7(3): 695704.

43. Ali BH (2004) Does gum Arabic have an antioxidant action in Rat kidney. Ren Fail 26(1): 1-3.

44. H Fujita, H Fujishima, S Chida, K Takahashi, Z Qi, et al. (2009) Reduction of renal superoxide dismutase in progressive diabetic nephropathy. J Am Soc Nephrol 20: 1303-1313.

45. BH Ali, I Al-Husseni, S Beegam, A Al-Shukaili, A Nemmar, et al. (2013) Effect of gum arabic on oxidative stress and inflammation in adenineinduced chronic renal failure in rats. PLoS One 8: e55242.

46. H Kong, J Yang, Y Zhang, Y Fang, K Nishinari, et al. (2014) Synthesis and antioxidant properties of gum arabic-stabilized selenium nanoparticles. Int J.Biol Macromol 65: 155-162.

47. EY Park, H Murakami, Y Matsumura (2005) Effects of the addition of amino acidsand peptides on lipid oxidation in a powdery model system. J Agric Food Chem 53: 8334-8341.

48. R Marcuse (1960) Antioxidative effect of amino-acids. Nature 186: 886887.

49. Y Liu, Z Hou, J Yang, Y Gao (2015) Effects of antioxidants on the stability of beta-Carotene in $\mathrm{O} / \mathrm{W}$ emulsions stabilized by Gum Arabic. J Food Sci Technol 52: 3300-3311.

50. RL Yang, YH Shi, G Hao, W Li, GW Le (2008) Increasing oxidative stress with progressive hyperlipidemia in human: relation between malondialdehydeand atherogenic index. J Clin Biochem Nutr 43: 154158.

51. PV Limaye, N Raghuram, S Sivakami (2003) Oxidative stress and gene expression of antioxidant enzymes in the renal cortex of streptozotocininduced diabetic rats. Mol Cell Biochem 243: 147-152.

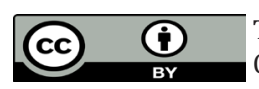

This work is licensed under Creative Commons Attribution 4.0 License

Submission Link: https://biomedres.us/submit-manuscript.php

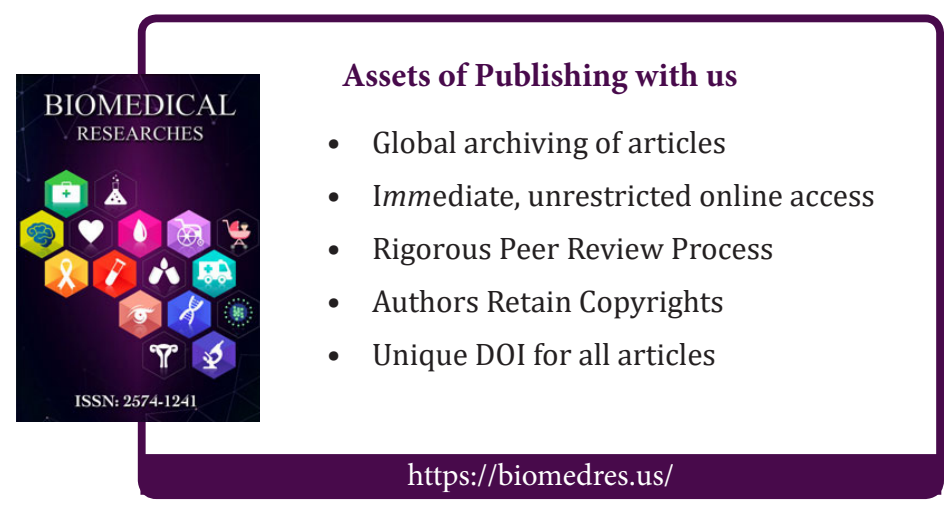

